\title{
A Review on Compressive Sensing for Distributed Signal Processing in WBAN
}

\author{
Ashwini S Chiwarkar \\ Department of Electronics \& \\ Telecommunication Engineering \\ Shri Sant Gajanan Maharaj College of \\ Engineering \\ Shegaon, Maharashtra, India
}

\author{
Dr. K. B. Khanchandani \\ Department of Electronics \& \\ Telecommunication Engineering \\ Shri Sant Gajanan Maharaj College of \\ Engineering \\ Shegaon, Maharashtra, India
}

\begin{abstract}
Wireless networks of the body (WBANs) that support healthcare applications are in the early stages of development but make valuable contributions to surveillance, diagnostics or therapy. They cover real-time medical information acquisition from various sensors with secure data communication and low power consumption. WBANs promises discreet outpatient medical monitoring over a long period of time and inform the physician in realtime about the patient's condition. They are widely used for ubiquitous healthcare, entertainment, and military applications. This article presents distributed wireless networks and describes the search for Orthogonal Matching Pursuit (OMP), Basis Pursuit (BP), Least Mean Square Technique (LMS), and Normalized Least Mean Square Technique (NLMS).
\end{abstract}

Keywords: WBAN, CS, OMP, BP, LMS, NLMS.

\section{INTRODUCTION}

In wireless networks, distributed signal processing methods are utilized for statistical inference. Information is extracted from data collected at spread nodes across a geographic area using distributed processing techniques. A set of neighboring nodes obtain their nearby data for each node, along with their local estimates for an improved estimate, and transmit their estimation to each node. Local information required for each node in distributed solutions. This method decreases the amount of processing required as well as the amount of bandwidth required for communication.

A WLAN network extends a WLAN hotspot across a larger geographical area without wires being connected to every access point (AP). It consists of two or more Wi-Fi (access points) base stations operating as a single system. The backbone will be used for future data communication in distributed wireless networks, as a backbone for laptops, cellular phones, sensors, actuators and computers. Nodes in distributed networks are directly connected to the network by at minimum 2 other nodes. There is no central controller in the distributed network which causes the whole network to collapse in this case. In addition, multiple routes for data transmission are available in a distributed network. Noise, interference, and other issues are reduced by using a distributed network. Information is extracted from data collected at nodes spread across a geographic area in dispersed wireless networks. In wireless distribution networks, training with other nodes is exchanged using the network topology and the parameters of interest are estimated.

Compressive sensing (CS) approaches have shown significant promise in signal compression to address the massive volume of information troubles, allowing for faster compression rates with reduced energy usage while maintaining the required distortion levels. First, the captured signals are compressed using a random matrix to project them onto a lower dimension. The compressed signals are subsequently transferred via the neighboring terminal and device. Finally, CS recovery techniques can be used to restore the original signal for processing and assessment. Traditional CS, on the other hand, concentrates on signals with weak patterns. In previous times considerable work has been done on modeling such source data using the structural characteristics of the signals to improve the performance and speed of CS reconstruction algorithms because of the growing number of source data that contain sparse or low-rank structures.

\section{LiteratURE REVIEW}

Brunelli and Caione [14] investigated the energy usage issue of both digital and analog CS, conducting a valid assessment utilizing real resource-constrained hardware architecture to investigate the influence of CS variable on signal recovery performance and sensor longevity. 
Majumdar and Ward [16] integrate state-of-the-art blind CS and low-rank approaches, and then create a Split Bregman strategy to address the EEG signal restoration issue in WBAN.

Wang et al. [18] discovered that the quantization module is an underappreciated but critical aspect in the total energy usage of the CS sampling process, and they went on to offer two illuminating adjustable quantized CS topologies for body sensor networks.

Furthermore, Li et al. [19] focused on developing a weighted group sparse generative model for signal retrieval at the fusion centre by capitalizing on the sparsity property of bio signals.

Dixon et al. [20] examine the systematic development and implementation of CS in ECG and electromyography (EMG) sensors.

Yizhou YANG [21] presented a long-term channel preview scheme based on LSTM WBAN. An online approach has been created to allow the suggested predictor to work continuously in order to handle genuine use scenarios. When compared to the benchmark Moving Average predictor, the LSTM predictor gave up to $2 \mathrm{~s}$ of prediction ahead with a $50 \%$ NMSE reduction when evaluated using empirical measurements. When sketched to an appropriate power management technique, reliability and power consumption improvements are noticeable compared to other predictive methods. Other WBAN resource allocation tasks, such as MAC scheduling and relay transmission, benefit from the prediction method.

\section{METHODOLOGY}

Compressive sensing (CS) is a technique for enabling realtime information transfer in a wireless networks by drastically reducing the amount of local computation and data sets that must be sent over wireless links to a remote fusion centre. The huge data volume of array signals enables WSAN, which is a constraint. When compared to typical data compression techniques. Compressive sensing (CS) advancements have led to innovation and creativity about how to develop energyefficient WSNs with minimal cost data gathering. For effective high-dimensional sparse signal capture, CS has considered as a leading paradigm. Compression, specifically, is a simple linear operation that is independent of signal characteristics and is accomplished using random projection matrices. Numerous reconstructing strategies have been developed to rebuild the original high-dimensional signal from its compressed counterpart, each of which differs in regards of recovery performance and computing complexity. For various reasons, CS is well-motivated for a range of WSN applications. Data compression proceeding to transmit inside WSNs is critical due to the fundamentally limited energy and communication resources in WSNs. Sparsity, on the other hand, is a common feature of many signals of interest, which can be detected in a variety of designs and aspects. Then as result, data collection with led to the reduced rate samples, as needed by several atmosphere and infrastructure tracking apps, is an instant usage of CS in WSNs. CS-based data collection can take advantage of temporal and/or spatial sparsity.

\section{A. Orthogonal Matching Pursuit (OMP)}

The most prominent greedy technique for finding a sparse solution vector to an unresolved linear system of equations is Orthogonal Matching Pursuit (OMP). To find the indices of the help of the sparse solution vector, OMP uses the projecting process. As an upgrade to Matching Pursuit, Orthogonal Matching Pursuit was created. As a result, it exhibits many of the same characteristics as Matching Pursuit. Orthogonal Matching Pursuit (OMP) uses the nomenclature to 'greedily' develop the set $\Gamma^{n}$, inserting a single component in each iteration. After upgrading $\Gamma^{n}$, algorithm use the following formula to estimate $\mathrm{x}$ :

$$
x \approx \widehat{x^{n}} \Phi_{\Gamma^{n}} S_{\Gamma^{n}}^{n}=\Phi_{\Gamma^{n}} \Phi_{\Gamma^{n}}^{\dagger} x
$$

Orthogonal Matching Pursuit constructs a new signal estimate $\widehat{x^{n}}$ for each iteration. Within next iteration, the estimate error $\Re^{n}=x-\widehat{x^{n}}$ is analysed to evaluate whichever new element should be chosen. The inner products of the current residual $\mathfrak{R}^{n}$ and the column vectors $\psi_{i}$ of $\Phi$ are used to make the decision. Allow these internal products to exist

$$
\alpha_{i}^{n}=\psi_{i}^{T} \mathfrak{R}^{n}
$$

The novel element is then chosen for which $\alpha_{i}^{n}$ has the maximum magnitude, i.e

$$
\begin{gathered}
i_{\max }^{n}=\arg _{i} \max \left|\alpha_{i}^{n}\right| \\
\text { Thus, } \Gamma^{n+1}=\Gamma^{n} \cup i_{\max }^{n}
\end{gathered}
$$

It is vital to understand that the OMP selection technique does not choose the element that reduces the remaining norm following orthogonal projection of the signal onto the different components.

The advantage of OMP is that it has a significantly quicker runtime bound. For any fixed signal, OMP operates with a maximum probability. It is possible to obtain the 99 $\%$ restoration trend. 
The assurances are not all the same. Instead of all signals, the probability is for a fixed signal. The sort of measurement matrix is likewise more restricted herein, and it is uncertain whether OMP works in the situation of random Fourier matrices in the important scenario.

Algorithm: CS recovering using OMP

Initialize:

$x \approx \widehat{x^{n}} \Phi_{\Gamma^{n}} S_{\Gamma^{n}}^{n}=\Phi_{\Gamma^{n}} \Phi_{\Gamma^{n}}^{\dagger} x$

OMP constructs a new signal estimate $\widehat{x^{n}}$ for each iteration

Next iteration, estimate error $\mathfrak{R}^{n}=x-\widehat{x^{n}}$

Decision making the column vectors $\psi_{i}$ of $\Phi$

$\alpha_{i}^{n}=\psi_{i}^{T} \mathfrak{R}^{n}$

$i_{\max }^{n}=\arg _{i} \max \left|\alpha_{i}^{n}\right|$

$\Gamma^{n+1}=\Gamma^{n} \cup i_{\max }^{n}$

\section{B. Basis Pursuit (BP)}

Multiple strategies for solving the sparse restoration challenge and thus its applicability have been developed through compressive sensing. We explored at Basis Pursuit, an approach for solving the sparse recovery challenge that uses a linear program. Due to recent improvements in linear programming, a technique of decomposing relies on a true global optimum is at least theoretically viable. They choose one of the numerous possible solutions to $\Phi_{\alpha}=S$ whose coefficients have the minimum $l^{1}$ norm.

$$
\min \|\alpha\|_{1} \text { subject to } \Phi_{\alpha}=S
$$

Each of the dictionaries is a compilation of signals $\left(\Phi_{\Upsilon}\right)_{\Upsilon \in \Gamma_{1}}$, and we have looked at them one by one. Consider a signal decomposing as:

$$
S=\sum_{\Upsilon \in \Gamma_{1}} \alpha_{\Upsilon} \Phi_{\Upsilon 1}
$$

or signal decomposing that is approximately equivalent to

$$
S=\sum_{\Upsilon \in \Gamma_{1}} \alpha_{\Upsilon} \Phi_{\Upsilon 1}+\Re_{1}
$$

They suggest approximation decomposition as in (7) for Now, output $\mathrm{Y}(\mathrm{n})$ can be expressed as: collecting and organizing information with noise levels $(\sigma)$ greater than zero.

$$
\min \left\|\Phi_{\alpha}-S\right\|_{2}^{2}+\lambda_{n}\|\alpha\|_{l 1}
$$

With $\lambda_{n}=\sigma \sqrt{2 \log (\# \mathbb{D})}$ depending on the amount of different vectors in the dictionary \# $\mathbb{D}$.

In compressive sensing, Basis Pursuit has a number of benefits over other algorithms. All sparse signals are reconstructed in Basis Pursuit. As a result, the assurances it provides are consistent, implying that the technique will not collapse for any sparse signal. Basis Pursuit is clearly accurate and stable. The existence of two specific frequencies is resolved by Basis Pursuit, but not by the other approaches.

Even though Basis Pursuit offers these solid guarantees, it does have one drawback: speed. It is based on linear programming, which has a polynomial runtime despite being typically fairly efficient in reality. As a result, significant compressive sensing research has been done with speedier approaches.

Algorithm: CS recovering using BP

Initialize: input sparse signal

Select one of the possible solutions to $\Phi_{\alpha}=S$

Coefficients having $1_{1}$ norm $\min \|\alpha\|_{1}$ subject to $\Phi_{\alpha}=S$

Compilation of signals into dictionary $\mathrm{D}=\left(\Phi_{\Upsilon}\right)_{\Upsilon \in \Gamma_{1}}$

$S=\sum_{\Upsilon \in \Gamma_{1}} \alpha_{\Upsilon} \Phi_{\Upsilon 1}+\Re_{1}$ Approximate equivalent decomposed signal

Decision making, noise level $\sigma>0$

$\min \left\|\Phi_{\alpha}-S\right\|_{2}^{2}+\lambda_{n}\|\alpha\|_{l 1}$ final output

end

\section{Least Mean Square Technique (LMS)}

The LMS algorithm, which is dependent on Wiener filtration theory, stochastic averaging, and the least squares method, is the star player in the category of stochastic gradient algorithms. Every iteration of the LMS algorithm necessitates three distinct steps, which must be completed in the following order:

Lets suppose the input of the adaptive filter is $X(n)$, scale vector is $W(n)$ and output is $Y(n)$, here firstly scalable vector of the filter is expressed as:

$$
\vec{W}(n)=\left[W_{0}(n) W_{1}(n) \ldots \ldots W_{N-1}(n)\right]^{\mathcal{T}}
$$

$$
Y(n)=\sum_{i=0}^{N-1} W_{i}(n) X(n-1)
$$

Now its time to discuss about the steps which we have discussed above 
Step 1 - Equation (10) is used to calculate the filter output y(n).

2. The expected error value is computed as $E(n)=D(n)-$ $Y(n)$

3. Equation (11) is used to modify the weights of the filter vector.

$$
\vec{W}(n+1)=\vec{W}(n)+2 \mu E(n) \vec{X}(n)
$$

The convergence constant, or step size, of the LMS method is variable M. A low positive constant has an impact on algorithm adaption qualities.

Many statistical software packages that do not offer maximum likelihood estimations may include non-linear least squares software. It has a broader use than maximal likelihood. That is, if your software supports non-linear fitting and allows you to define the probability function you want to use, you can calculate least squares estimates for that distribution.

It is not easily applicable to data that has been suppressed. In comparison to maximum likelihood, it is often thought to have less desirable optimality qualities. The choice of initial values can have a big impact.

\section{Algorithm: CS recovering using LMS}

Initialize:

input of the adaptive filter is $\mathrm{X}(\mathrm{n})$ and scalable vector of the filter

$\vec{W}(n)=\left[W_{0}(n) W_{1}(n) \ldots \ldots W_{N-1}(n)\right]^{\mathcal{T}}$

Output of the filter

$Y(n)=\sum_{i=0}^{N-1} W_{i}(n) X(n-1)$

Compute error value $E(n)=D(n)-Y(n)$

Step size decided is $M$

Modify weight of filter vector

$$
\vec{W}(n+1)=\vec{W}(n)+2 \mu E(n) \vec{X}(n)
$$

end

end

\section{Normalized Least Mean Squares Algorithm (NLMS)}

The Normalized Least Mean Square (NLMS) algorithm is a gradient class adaptive technique that solves the Least Mean Square (LMS) algorithm's sluggish convergence problem. When the input signal $\mathrm{x}(\mathrm{n})$ has relatively high values, the LMS algorithm causes the noise to be amplified. For every iteration of the normalized LMS (NLMS) method, a variable convergence constant is computed utilizing the input signal power.

$$
\begin{gathered}
\vec{W}(n+1)=\vec{W}(n)+\frac{\mu}{\|\vec{X}(n)\|} \vec{X}(n) E(n) \\
\mu_{n}=\frac{\mu}{\|\vec{X}(n)\|^{2}}
\end{gathered}
$$

The NLMS algorithm's practical implications is extremely similar to the LMS algorithms since it is an expansion of the ordinary LMS method.

The NLMS technique is less computationally intensive and has a fast convergence rate, making it ideal for echo cancellation. With uncertain input signals, it is more stable.

Due to the existence of normalized step size, the noise amplification shrinks or shrinks in size. It has the smallest study state error and the quickest convergence.

\section{Algorithm: CS recovering using NLMS}

Initialize: input signal $\mathrm{x}(\mathrm{n})$

For every iteration compute the value of convergence constant

$\vec{W}(n+1)=\vec{W}(n)+\frac{\mu}{\|\vec{X}(n)\|} \vec{X}(n) E(n)$

$\mu_{n}=\frac{\mu}{\|\vec{X}(n)\|^{2}}$

end

end

\section{CONCLUSION}

The popularity of wearable devices is revolutionizing traditional medical models. WBAN can not only free people from traditional hospitals and clinics, but more importantly, reduce the burden of disease management for people with chronic conditions such as diabetes and hypertension. This paper presents distributed wireless networks and the methodology describes Orthogonal Matching Pursuit (OMP), Basis Pursuit (BP), Least Mean Square Technique (LMS), and Normalized Least Mean Square Technique (NLMS.

\section{REFERENCES}

[1] Zhang, YB., Huang, LT., Li, YQ. et al. "Low-rank and joint-sparse signal recovery using sparse Bayesian learning in a WBAN", Multidim Syst Sign Process 32, 359-379 (2021). https://doi.org/10.1007/s11045020-00743-y.

[2] G. Mateos, I. D. Schizas, and G. B. Giannakis, "Distributed Recursive Least- Squares for Consensus-Based In-Network Adaptive Estimation," IEEE Trans. Signal Process., vol. 57, no. 11, pp. 45834588, November 2009.

[3] C. G. Lopes and A. H. Sayed, "Incremental adaptive strategies over distributed networks," IEEE Trans. Signal Process., vol. 48, no. 8, pp. 223-229, August 2007. 
[4] A. H. Sayed and C. G. Lopes, "Adaptive processing over distributed networks," IEICE Trans. Fundam. Electron. Commun. Comput. Sci., vol. E90-A, no. 8, pp. 1504-1510, August 2007.

[5] Brunelli D and Caione C. Sparse recovery optimization in wireless sensor networks with a sub-Nyquist sampling rate. Sensors 2015; 15(7): 16654-16673.

[6] Majumdar A and Ward RK. Energy efficient EEG sensing and transmission for wireless body area networks: a blind compressive sensing approach. Biomed Signal Proces 2015; 20: 1-9.

[7] Wang A, Lin F, Jin Z, et al. A configurable energy efficient compressive sensing architecture with its application on body sensor networks. IEEE T Ind Inform 2016; 12(1): 15-27.

[8] Li S, Da Xu L and Wang X. A continuous biomedical signal acquisition system based on compressive sensing in body sensor networks. IEEE T Ind Inform 2013; 9(3): 1764-1771.

[9] Dixon AM, Allstot EG, Gangopadhyay D, et al. Compressive sensing system considerations for ECG and EMG wireless biosensors. IEEE T Biomed Circ S 2012; 6(2): 156-166.

[10] Yang, Y., Smith, D. B., \& Seneviratne, S. (2019). Deep Learning Channel Prediction for Transmit Power Control in Wireless Body Area Networks. IEEE International Conference on Communications, 2019May, 1-6. https://doi.org/10.1109/ICC.2019.8761432 